\title{
STAT3 mediates resistance to anoikis and promotes invasiveness of nasopharyngeal cancer cells
}

\author{
CHIEN-HUNG LIN ${ }^{1,2}$, MING-CHANG CHIANG ${ }^{3}$ and YANN-JANG CHEN ${ }^{1,4,5}$ \\ ${ }^{1}$ Institute of Clinical Medicine, National Yang-Ming University, Taipei 112; \\ ${ }^{2}$ Department of Pediatrics, Zhongxing Branch, Taipei City Hospital, Taipei 10341; ${ }^{3}$ Department of Life Science, \\ College of Science and Engineering, Fu Jen Catholic University, New Taipei City 24205; ${ }^{4}$ Department of Life Sciences \\ and Institute of Genome Sciences, National Yang-Ming University, Taipei 112; ${ }^{5}$ Department of Pediatrics, \\ Renai Branch, Taipei City Hospital, Taipei 10629, Taiwan, R.O.C.
}

Received November 9, 2016; Accepted September 8, 2017

DOI: $10.3892 /$ ijmm.2017.3151

\begin{abstract}
Nasopharyngeal carcinoma (NPC), a tumor arising from the epithelial cells of the nasopharynx, is endemic in Southeast Asia and Taiwan. The prognosis of NPC patients with local recurrence and metastasis is poor. Resistance to anoikis is a primary characteristic of tumor cells that metastasize. However, the mechanism through which NPC cells resist anoikis and are able to metastasize has not been fully elucidated. In the present study, the acquisition of anoikis resistance was analyzed in the TW01 and TW06 human NPC cell lines growing under anchorage-independent conditions. A considerable number of TW01 and TW06 cells was found to be resistant to anoikis and exhibited a higher capability of migration and invasion. These anoikis-resistant NPC cells exhibited significantly increased expression of signal transducer and activation of transcription 3 (STAT3) compared with adherent cells. Furthermore, blockade of STAT3 expression by STAT3 inhibitors or STAT3 silencing significant increased anoikis in anoikis-resistant NPC cells. Moreover, silencing STAT3 not only reduced the capacity of NPC cells to resist anoikis, but also reversed their invasive properties. The expression of epithelial-to-mesenchymal transition-related proteins and CD44 was also significantly decreased following STAT3 knockdown. The results of the present study established that STAT3 mediates anoikis resistance, with enhanced cell migration and invasion of NPC cells, and that activation of STAT3 may increase metastatic capacity, indicating the crucial role of
\end{abstract}

Correspondence to: Dr Chien-Hung Lin, Department of Pediatrics, Zhongxing Branch, Taipei City Hospital, 145 Zhengzhou Road, Datong, Taipei 10341, Taiwan, R.O.C.

E-mail:dan08@tpech.gov.tw

Dr Yann-Jang Chen, Department of Life Sciences and Institute of Genome Sciences, National Yang-Ming University, No. 155, Section 2, Linong Street, Taipei 112, Taiwan, R.O.C.

E-mail: yjchen@ym.edu.tw

Key words: nasopharyngeal carcinoma, signal transducer and activator of transcription 3 , anoikis, neoplasm invasiveness
STAT3 in conferring anoikis resistance and enhanced invasive properties to NPC cells.

\section{Introduction}

Nasopharyngeal carcinoma (NPC), a tumor arising from nasopharyngeal epithelial cells, is one of the most common head and neck cancers in Southeast Asia and Taiwan. Lymph node and distant organ metastasis is a major challenge in NPC, as it is often responsible for treatment failure $(1,2)$. Although radiotherapy, alone or combined with chemotherapy, has been traditionally used as the treatment of choice for NPC, a significant number of patients with advanced NPC develop metastasis and local recurrence. Therefore, in order to establish more effectively targeted therapies, the mechanisms underlying resistance development and metastasis of NPC must be elucidated.

Epithelial cell adhesions may allow survival of cancer cells and facilitate tumor growth. However, some tumor cells develop properties that enable them to survive under anchorage-independent conditions and continue to proliferate $(3,4)$. These cells are referred to as being resistant to anoikis, and they are able to invade easily and migrate to distant metastatic sites (5). The mechanism underlying tumor cells acquiring anoikis resistance and enhanced migratory ability remains largely unexplored. However, it is crucial to elucidate why NPC cells gain metastatic potential following resistance to anoikis.

Signal transducer and activator of transcription 3 (STAT3) is an oncogenic transcription factor that mediates cellular response to several growth factors and cytokines. Increased expression of STAT3 is involved in the control of cell growth and has been associated with inflammation, survival, proliferation and angiogenesis (6-8). In addition, activation of STAT3 was detected in the development and progression of various tumors, and this pathway was found to be a potential target for cancer treatment (9). Phosphorylation of STAT3 enhances the expression of a variety of proliferation and survival genes, such as cell survival-related genes (cyclin D1 and survivin), and anti-apoptotic proteins (Bcl-2 and Bcl-xL) (10,11). It has been reported that activation of STAT3 may directly contribute to the invasiveness of NPC cells $(12,13)$. However, the association between STAT3 expression and anoikis resistance, which may result in increased NPC aggressiveness, 
has not been clearly determined. The aim of the present study was to evaluate the potential role of STAT3 in anoikis resistance, and investigate whether the activation of STAT3 may lead to tumor progression and invasion in NPC.

\section{Materials and methods}

Cell culture. Two human NPC cell lines, TW01 and TW06, were cultured in $10-\mathrm{cm}^{2}$ dishes with Dulbecco's modified Eagle's medium (DMEM; Gibco/Thermo Fisher Scientific, Carlsbad, CA, USA) and 10\% fetal bovine serum (FBS; Bioind, Kibbutz Beit Haemek, Israel), 1\% sodium pyruvate (Bioind), $1 \%$ penicillin, streptomycin, amphotericin (PSA; Bioind), and $1 \%$ non-essential amino acids (NEAA; Bioind). The cells were incubated at $37^{\circ} \mathrm{C}$ in a humidified atmosphere of $95 \%$ air and $5 \% \mathrm{CO}_{2}$.

Migration and invasion assay. The cells were incubated under either anchorage-dependent or -independent conditions for $48 \mathrm{~h}$, and were then seeded in 6-well plates and grown to confluence. Analysis of cell migration was performed by the wound healing method. Cell monolayers were scraped with a 10- $\mu 1$ tip and photographed at the beginning of the assay $(0 \mathrm{~h})$ and at $24 \mathrm{~h}$ under the respective conditions. The invasion assay was performed by the Transwell system with a polycarbonate filter membrane (24-well insert, pore size $8 \mu \mathrm{m}$; Corning Costar, Corning, NY, USA). Each well was coated with Matrigel (60 $\mu \mathrm{g}$; BD Biosciences, San Diego, CA, USA) immediately prior to the invasion assay. After a 48-h incubation under adherent or suspension conditions, the cells were transferred to the upper chamber in a medium without serum or growth factors, and the medium supplemented with serum was used as a chemoattractant in the lower chamber. The cells were incubated for $48 \mathrm{~h}$, and the cells on the lower surface of the membrane were fixed with methanol and stained with crystal violet. The cells invading the membrane were counted under a light microscope (magnification, x100, 5 random fields/well).

Measurement of cells resistance to anoikis. TW01 and TW06 cells in the culture plate were detached and made into a single-cell suspension in serum-free DMEM/F12, and then seeded into a poly-HEMA-coated 6-well plates at a density of $1.5 \times 10^{5}$ cells $/ \mathrm{ml}$ for $48 \mathrm{~h}$. The cells were harvested and apoptosis was evaluated by flow cytometry using an Annexin V-FITC/propidium iodide kit (BD Pharmingen, San Diego, CA, USA). Anoikis resistance was calculated as percentage compared with control.

Antibodies and reagents. Antibodies against STAT3 (cat. no. S5933), phosphorylated STAT3 (tyrosine 705; cat. no. S4933), E-cadherin (cat. no. U3254), N-cadherin (cat. no. SAB2702400) and vimentin (cat. no. V6389), were purchased from Sigma-Aldrich (St. Louis, MO, USA). Antibodies against Bcl-2 (cat. no. sc-492) and survivin (cat. no. sc-17779) were purchased from Santa Cruz Biotechnology, Inc. (Santa Cruz, CA, USA). The STAT3 inhibitor Stattic was obtained from Selleck Chemicals (Boston, MA, USA). Stock solutions were prepared and stored according to the manufacturer's guidelines.
STAT3 blockade with shRNA transfection. Two STAT3 shRNAs with green fluorescent protein (GFP) obtained from Qiagen (Valencia, CA, USA) (cat. no. 336311) were used to knock down STAT3 as shRNA1 and shRNA2. Transfections in TW01 and TW06 cells were performed using PolyJet ${ }^{\mathrm{TM}}$ reagent (SL100688; SignaGen ${ }^{\circledR}$ Laboratories, Rockville, MD, USA) according to the manufacturer's protocol. After $24 \mathrm{~h}$ of transfection, the cells were processed for anoikis assay, invasion assay or western blotting, as described above. Prior to the experiment, the extent of silencing was tested by western blotting.

Western blot analysis. TW01 cells were incubated in either anchorage or suspension culture, with or without any treatment, for $48 \mathrm{~h}$. The cells were then collected and lysed using RIPA buffer (Thermo Fisher Scientific Inc., Rockford, IL, USA) containing protease inhibitors. Samples with $20 \mu \mathrm{g}$ of protein were subjected to sodium dodecyl sulfate-polyacrylamide gel electrophoresis, transferred onto a polyvinylidene difluoride membrane (Millipore, Bedford, MA, USA), followed by blocking with $5 \%$ milk in Tris-buffered saline (TBS). After blocking, the membranes were immunoblotted with the specific antibodies and incubated in TBS at $4^{\circ} \mathrm{C}$ overnight. Primary antibodies against STAT3 (rabbit polyclonal), phosphorylated STAT3 (tyrosine 705; rabbit polyclonal), E-cadherin (rat monoclonal), N-cadherin (rabbit polyclonal) and vimentin (mouse monoclonal) were used at a dilution of 1:1,000. Primary antibodies against Bcl-2 (rabbit polyclonal) and survivin (mouse monoclonal) were used at a dilution of 1:200. A rabbit polyclonal anti-GAPDH (cat. no. G9545; Sigma-Aldrich) was used as sample-loading control at a dilution of 1:1,000. The membranes were then washed three times with TBST (1X TBS, $0.1 \%$ Tween20), and then incubated with goat anti-rabbit (cat. no. ab205718, Abcam, Cambridge, UK), goat anti-mouse (cat. no. ab205719, Abcam), or goat anti-rat (cat. no. ab97057, Abcam) secondary antibodies conjugated with horseradish peroxidase at a dilution of 1:5,000 in TBS for $1 \mathrm{~h}$ at room temperature. The membranes were washed again in TBST three times at room temperature. Immunoreactive protein bands were detected with the enhanced chemiluminescence detection system (GE Healthcare, Life Sciences, Pittsburgh, PA, USA).

Flow cytometry for anti-CD44 antibody analysis. The presence of CD44 on the cell surface was detected using anti-CD44-FITC (BD Biosciences) antibody. The dissociated cells were stained with anti-human CD44 and were then incubated at $4^{\circ} \mathrm{C}$ for $15 \mathrm{~min}$ in the dark. Following incubation, the cells were washed once with cold FACS buffer. The labeled cells were analyzed by Gallious Flow Cytometry (Beckman Coulter, Brea, CA, USA) and the data were analyzed with FlowJo software (Tree Star Inc., Ashland, OR, USA).

\section{Results}

A considerable number of NPC cells exhibit anoikis resistance. Anoikis resistance was evaluated in TW01 and TW06 NPC cells, which were cultured under anchorage-independent conditions for $48 \mathrm{~h}$. Cell survival was assessed by flow cytometry and compared with the cells simultaneously cultured under adherent conditions. Although anoikis was induced to a certain extent in tumor cells under anchorage-independent 

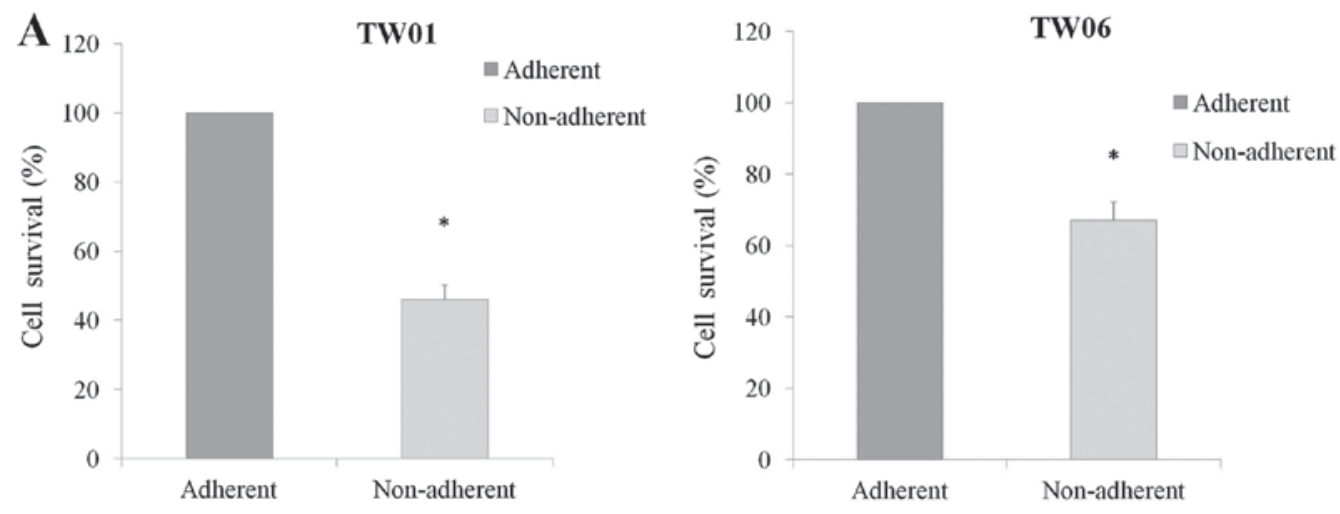

B

TW01

TW06

Adherent
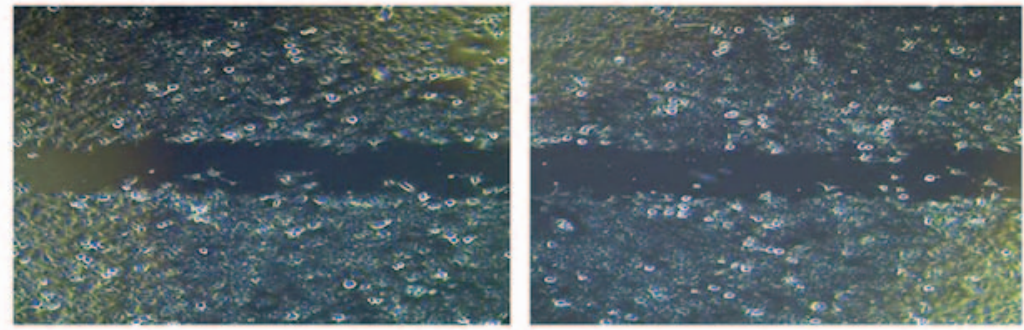

Anchorage-
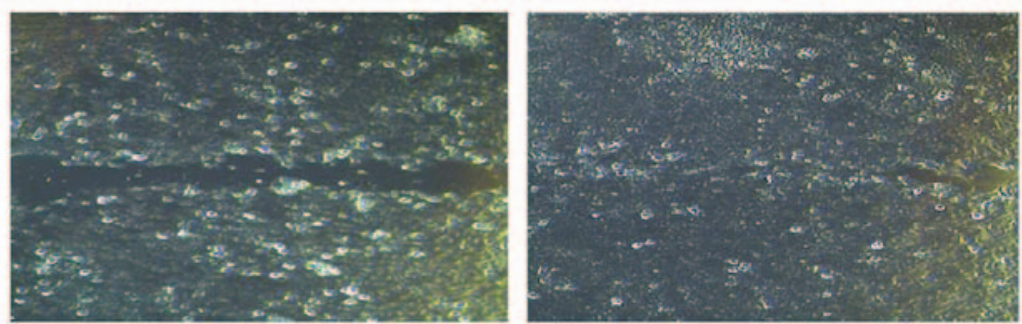

C
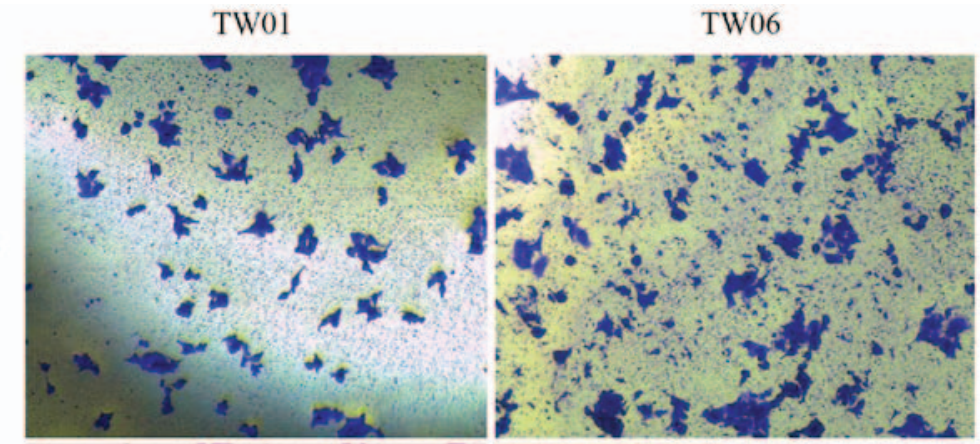

Adherent

Anchorage-

independent

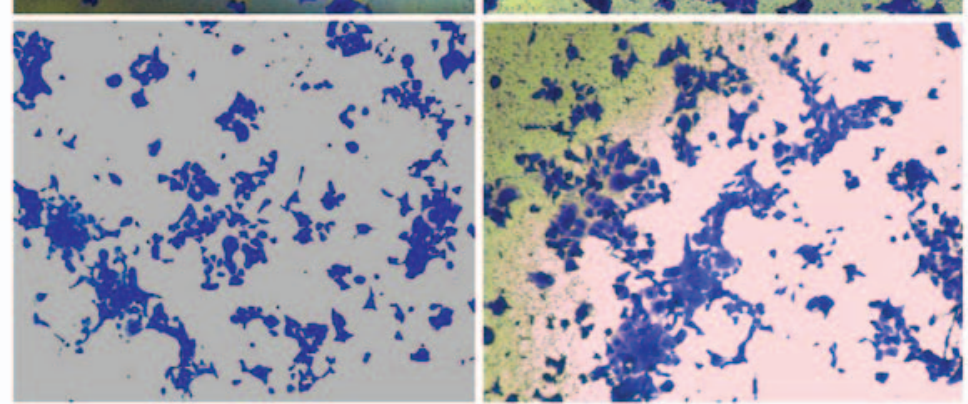

Figure 1. A significant number of nasopharyngeal carcinoma cells resisted anoikis under anchorage-independent conditions. (A) The survival percentage of anchorage-independent cells was compared with that of cells cultured under adherent conditions. (B) Wound healing assay performed on anoikis-resistant cells and compared with the adherent cells. (C) Transwell invasion assay performed on anoikis-resistant cells and compared with the adherent cells. Migrated cells, which had invaded into the lower chamber, were fixed with ethanol for $30 \mathrm{~min}$ and stained with $0.1 \%$ crystal violet for 10 min (magnification, x100).

conditions, a significant percentage of NPC cells resisted anoikis. A total of $\sim 46 \%$ of TW01 cells resisted anoikis, whereas $67 \%$ of TW06 cells resisted anoikis when cultured under anchorage-independent conditions (Fig. 1A). 

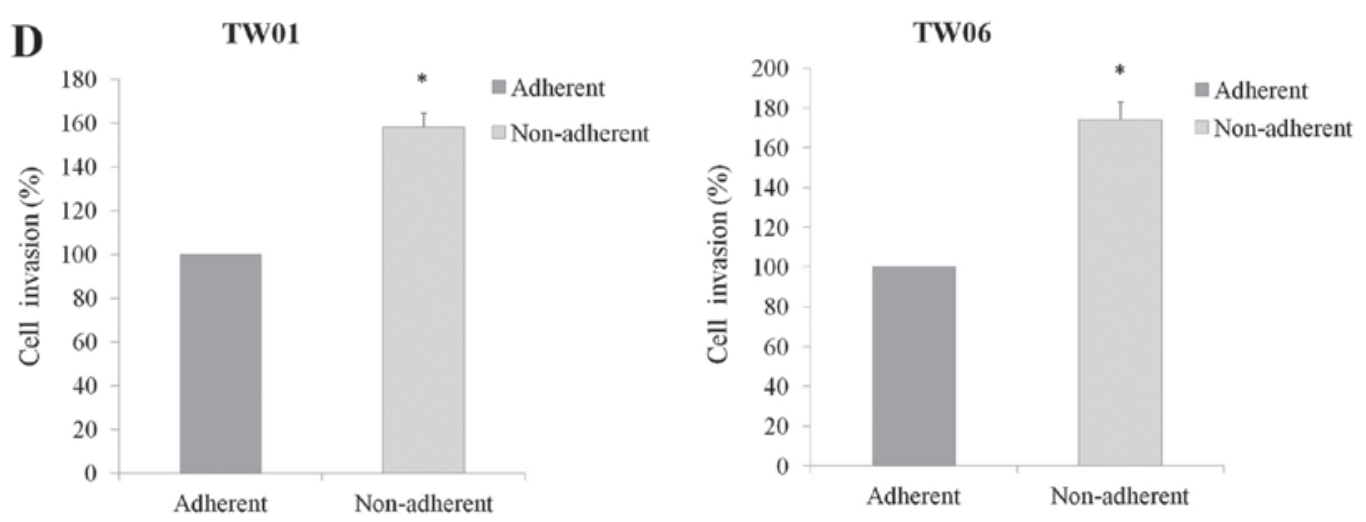

Figure 1. Continued. (D) The cells invading the membrane were counted under a light microscope (magnification, x100, 5 random fields/well). The values are plotted as mean \pm standard deviation. ${ }^{*} \mathrm{P}<0.05$ compared with the adherent group. Each experiment was repeated at least three times, with similar results.

Adherent

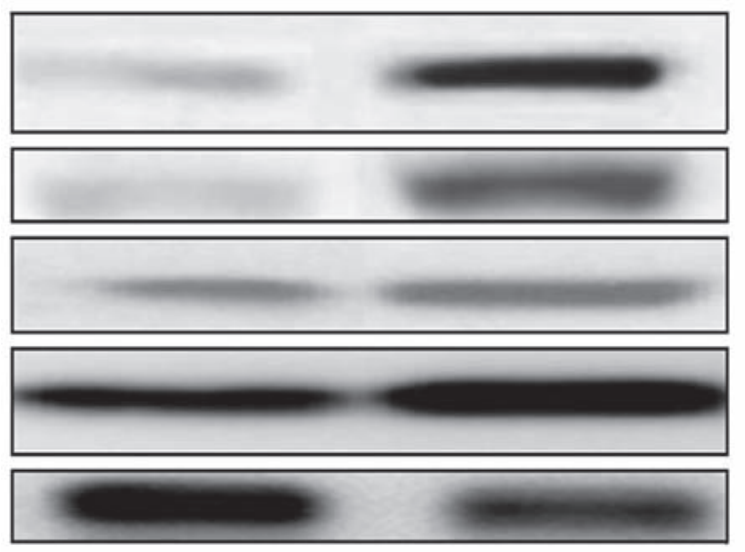

STAT3

p-STAT3

Bcl-2

Survivin

GAPDH

Figure 2. Representative western blot analyses of signal transducer and activation of transcription 3 (STAT3), p-STAT3, B-cell lymphoma-2 (Bcl-2) and survinin from anoikis-resistant and adherent cells. Glyceraldehyde 3-phosphate dehydrogenase (GAPDH) was used as the loading control.

Anoikis-resistant NPC cells exhibit a high capability of migration and invasion. Migration and invasion assays were performed to compare the metastatic potential of anoikis-resistant cells with that of adherent cells. The wound healing assay was used to evaluate cell migration in TW01 and TW06 cells. The cells were incubated under anchorage or anchorage-independent conditions for $48 \mathrm{~h}$ and then transferred to 6-well plates, and a wound was created when the cells were attached and started to grow. The results demonstrated that TW01 and TW06 cells that resisted anoikis healed the wound at a more rapid rate compared with adherent cells (Fig. 1B).

The invasion capacity of NPC cells was evaluated with the Transwell invasion assay. TW01 and TW06 cells that resisted anoikis were also found to be highly invasive compared with adherent cells (Fig. 1C). Anoikis-resistant TW01 cells exhibited a 58\% increased rate of invasion, whereas TW06 cells exhibited a $74 \%$ increased rate of invasion compared with their respective adherent cells (Fig. 1D). These results suggested that anoikis-resistant cells acquire enhanced migratory and invasive properties.

Overexpression of STAT3 in anoikis-resistant NPC cells. To better understand the molecular changes that are associated with
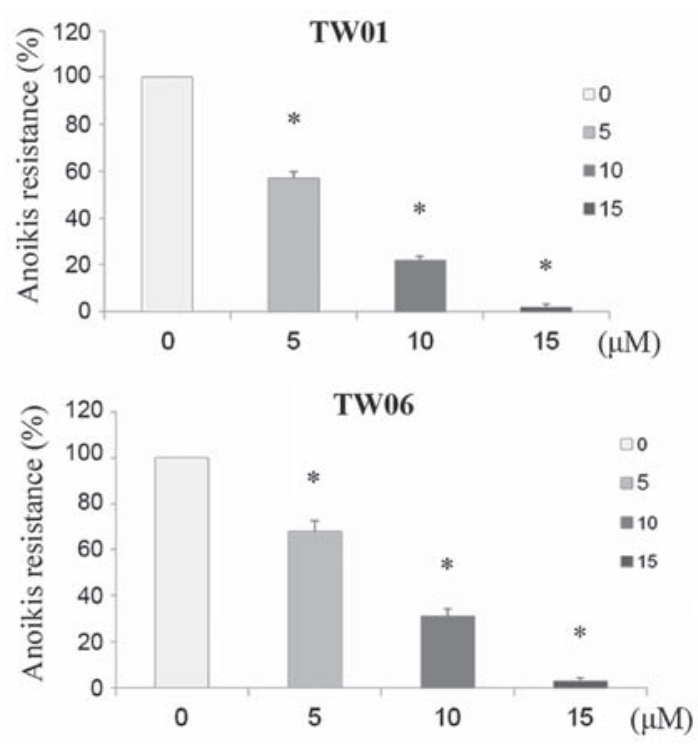

Figure 3. Effects of signal transducer and activation of the transcription 3 (STAT3) inhibitor Stattic on the sensitivity of anoikis-resistant nasopharyngeal carcinoma cells growing anchorage-independently. TW01 and TW06 cells were treated with different concentrations of Stattic for $48 \mathrm{~h}$ under anchorage-independent conditions. Following treatment, the proportion of anoikis-resistant cells was measured among these cells. Representative bar graphs show the percentage of anoikis-resistant cells under different treatment conditions. Values are plotted as mean \pm standard deviation. ${ }^{*} \mathrm{P}<0.05$ compared with the control group.

anoikis resistance and a highly migratory and invasive phenotype in NPC cells, the expression levels of proteins, including STAT3, phosphorylated STAT3 (p-STAT3), B-cell lymphoma-2 (Bcl-2) and survivin, was examined in TW01 anoikis-resistant and adherent cells by western blot analysis. The results demonstrated a significant increase in STAT3 and p-STAT3 in the cells that resisted anoikis compared with adherent cells. In addition, in anoikis-resistant cells, a significant increase was observed in the expression of the anti-apoptotic proteins Bcl-2 and survivin, which are regulated by STAT3 (Fig. 2).

STAT3 inhibitors overcome anoikis resistance in NPC cells. To determine whether STAT3 plays a significant role in anoikis resistance as well as in increased migratory and invasive potential leading to metastasis, the effect of the STAT3 inhibitor Stattic on anoikis resistance was evaluated in NPC 

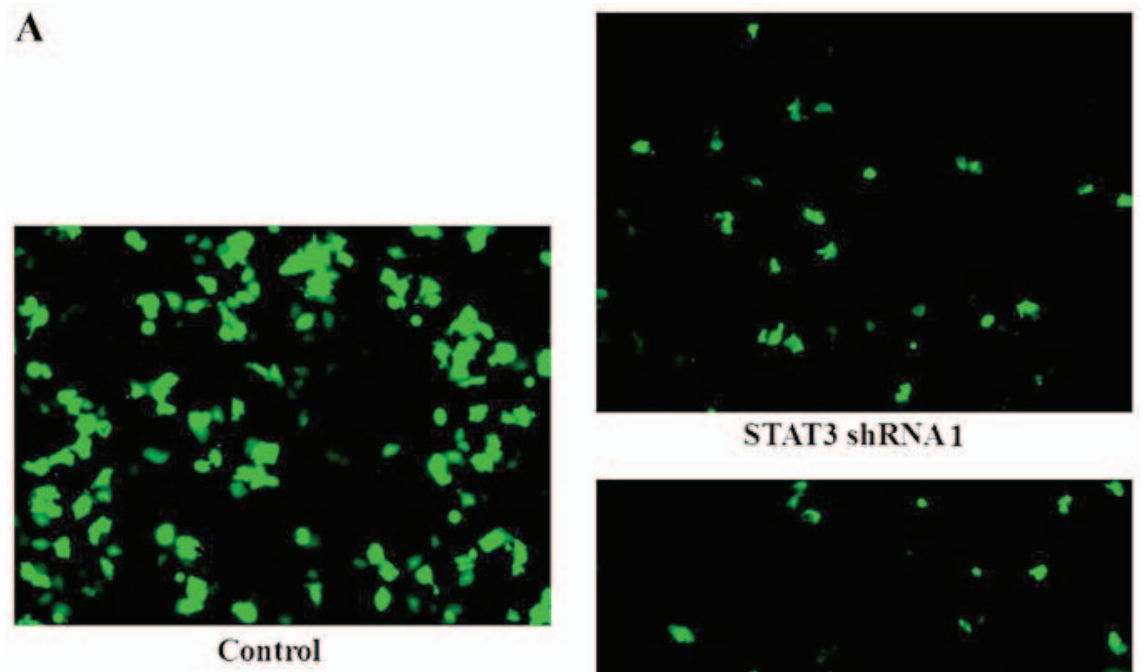

STAT3 shRNA1

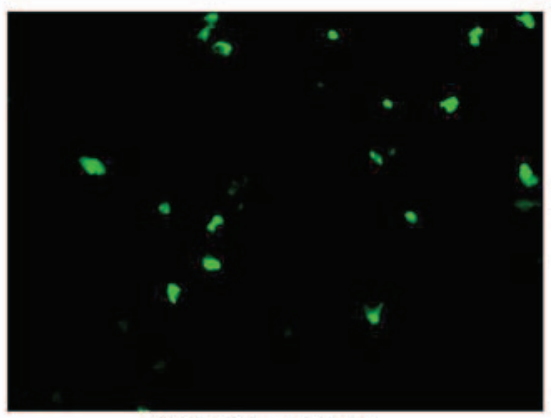

STAT3 ShRNA2
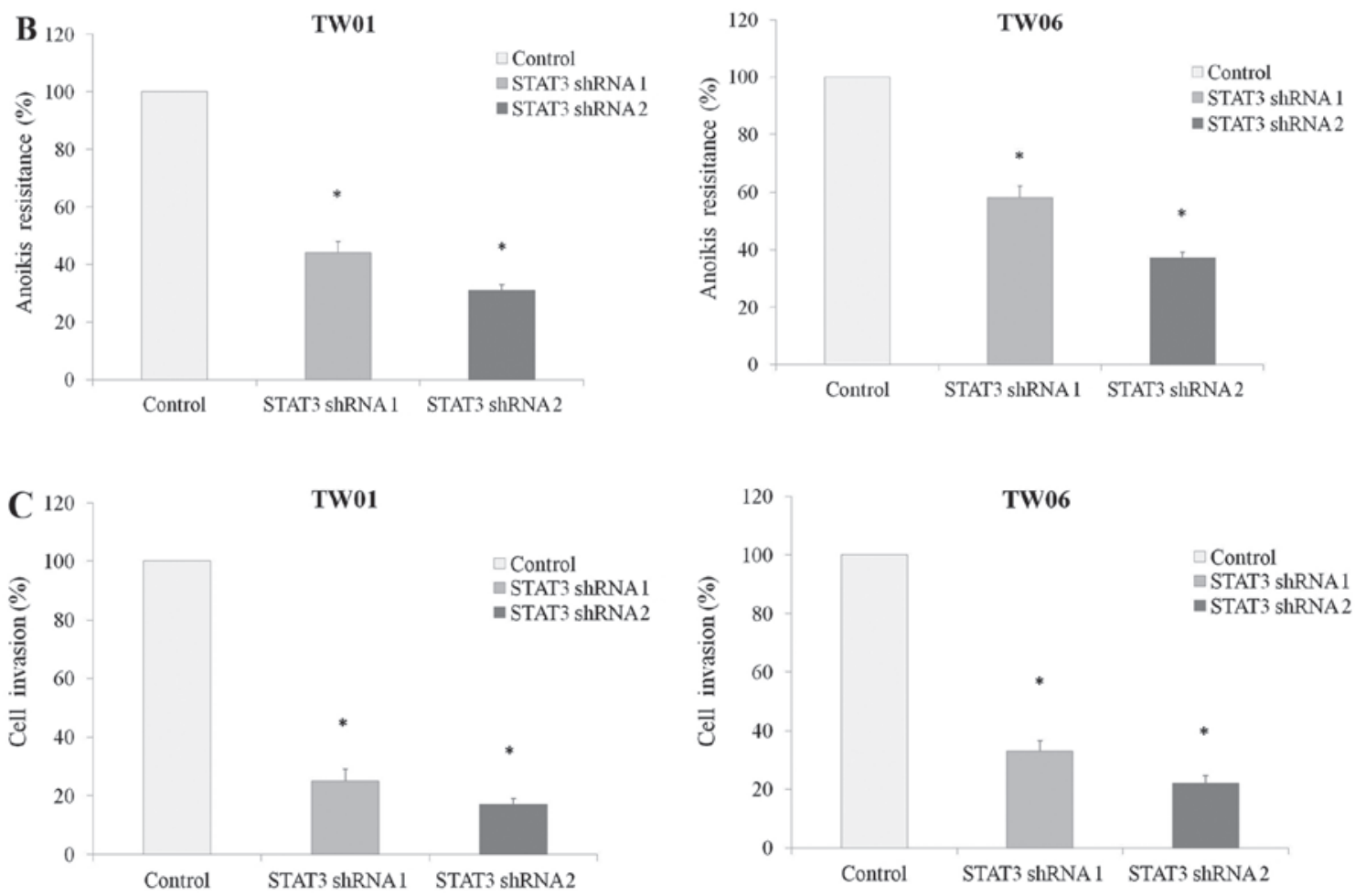

Figure 4. Effects of signal transducer and activation of transcription 3 (STAT3) silencing on the sensitivity and invasive capacity of of anoikis-resistant nasopharyngeal carcinoma (NPC) cells growing anchorage-independently. TW01 and TW06 cells were transfected with either STAT3 shRNA1 or shRNA2 for $24 \mathrm{~h}$, after which time they were cultured under suspension conditions for $48 \mathrm{~h}$. Cells transfected with scrambled shRNA and cultured under the same conditions were used as control. (A) The extent of the expression of STAT3-green fluorescent protein in cells transfected with scrambled shRNA STAT3 (control), STAT3 shRNA1 and STAT3 shRNA2 was direct visualized by fluorescence microscopy. (B) Following incubation for 48 h, TW01 and TW06 cells resistant to anoikis were measured and normalized against the control group. Values are plotted as mean \pm standard deviation (SD). "P<0.05. (C) The invasion assay was performed by the Transwell system with a polycarbonate filter membrane coated with Matrigel. Each experiment was repeated at least three times, with similar results. Values are plotted as mean \pm SD. ${ }^{*} \mathrm{P}<0.05$.

cells. Following treatment with $0,5,10$ and $15 \mu \mathrm{M}$ Stattic under suspension conditions for $48 \mathrm{~h}$, TW01 and TW06 cells were re-cultured on adherent plates. A significant reduction was observed in anoikis resistance following Stattic treatment in these NPC cells. Treatment with $5 \mu \mathrm{M}$ Stattic reduced anoikis resistance by $43 \%$ in TW01 and by 32\% in TW06 cells. The growth inhibition was increased by 78 and $69 \%$ following Stattic treatment at 5 and $10 \mu \mathrm{M}$, respectively (Fig. 3). Both 
A
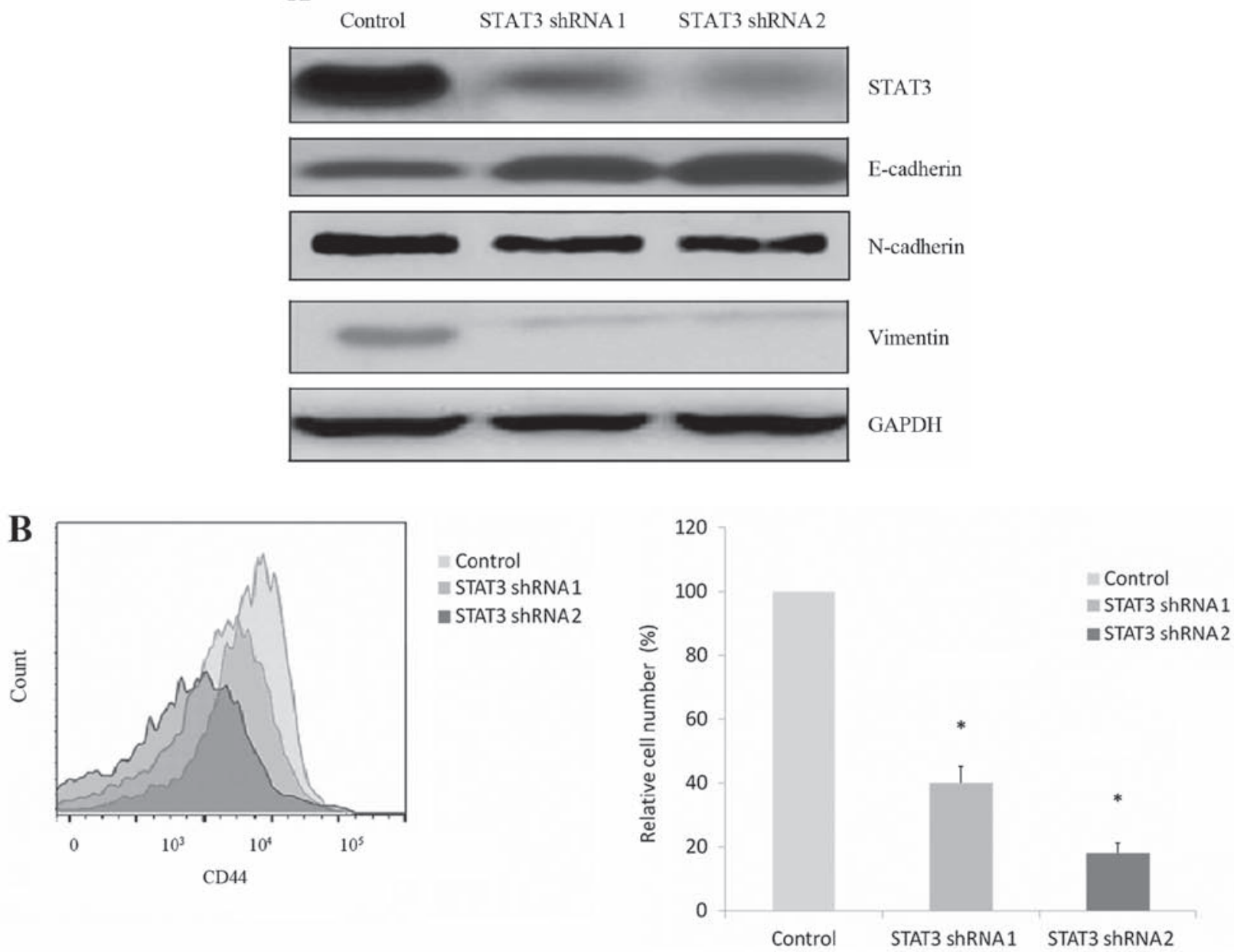

Figure 5. The protein levels of the epithelial-mesenchymal transition-related markers expression including E-cadherin, N-cadherin and vimentin was analyzed by western blot analysis and CD44 expression was detected by flow cytometry. (A) Expression of E-cadherin was significantly increased, but N-cadherin and vimentin were significantly decreased after signal transducer and activation of transcription 3 (STAT3) silencing. (B) CD44 expression was significantly decreased in STAT3 shRNA-transfected cells when compared to non-specific shRNA-transfected cells.

cell lines exhibited a $>90 \%$ reduction in anoikis following treatment with $15 \mu \mathrm{M}$ Stattic.

Interference with STAT3 expression blocks anoikis resistance in NPC cells. shRNA-mediated knockdown of STAT3 in TW01 and TW06 cells was further performed to establish the role of STAT3 in anoikis-resistant NPC cells. Two STAT3 shRNAs (shRNA1 and shRNA2) and scrambled shRNA as control were used for STAT3 knockdown. The STAT3-transfected cells expressing GFP were processed for fluorescence microscopy (Fig. 4A) and constitutively stable cells were used in the following experiments. The sensitivity to anoikis was further calculated and the result demonstrated a significant decrease in anoikis resistance as a result of STAT3 knockdown (Fig. 4B). Knockdown of STAT3 expression confers a 55-75\% reduction of anoikis resistance in TW01 cells and a 40-65\% reduction in TW06 cells. Our results demonstrated that the percentage of anoikis-resistant cells was highly correlated with the level of STAT3 expression.

Silencing STAT3 diminishes the invasiveness of anoikis-resistant NPC cells. Since the results demonstrated that anoikis resistance was associated with enhanced invasiveness, the
Transwell invasion assay was performed in TW01 and TW06 cells after silencing STAT3. There was a significant decrease in invasion after STAT3 silencing in these tumor cells, and the extent of decreased invasion was correlated with the level of STAT3 expression (Fig. 4C). STAT3 silencing contributed to a $75-85 \%$ decrease in the rate of invasion of TW01 cells, and there was a $\sim 65-80 \%$ decrease in the rate of invasion of TW06 cells after STAT3 silencing. Therefore, inhibition of STAT3 expression in TW01 and TW06 cells that have acquired anoikis resistance not only increased sensitivity to anoikis, but also interfered with their invasion capacity.

Silenced STAT3 decreases epithelial-to-mesenchymal transition (EMT) markers and CD44 expression. As changes in invasive properties were significantly correlated with STAT3 expression of anoikis-resistant NPC cells, the effect of STAT3 knockdown on the expression of migration and invasion regulatory proteins was further examined by western blot analysis and CD44 expression by flow cytometry. The levels of expression of EMT-related proteins, including E-cadherin, $\mathrm{N}$-cadherin and vimentin, was analyzed by western blot analysis and CD44 expression was detected by flow cytometry in anoikis-resistant TW01 cells with and without STAT3 silencing. 
Our data indicated that knockdown of STAT3 significantly increased E-cadherin, but decreased $\mathrm{N}$-cadherin and vimentin expression, as evaluated by western blot analysis (Fig. 5A). In addition, the results of flow cytometry demonstrated that CD44 expression was significantly decreased in STAT3 shRNA-transfected TW01 cells compared with non-specific shRNA-transfected TW01 cells (Fig. 5B).

\section{Discussion}

NPC is associated with a high incidence of recurrence and frequent initial dissemination to regional lymph nodes, representing a major challenge in clinical practice. Although radiotherapy and chemotherapeutic agents have been used in the management of NPC, these tumors usually recur and metastasize following treatment $(14,15)$. Anoikis is apoptosis that occurs in anchorage-dependent cells when they detach from the surrounding extracellular matrix, and it has been suggested that cancer cells develop different strategies for overcoming anoikis. Resistance to anoikis may enable tumor cells to metastasize and facilitate tumor formation in other sites $(16,17)$. However, the mechanisms underlying anoikis resistance in NPC cells, which may lead to a high rate of metastasis, have not been fully elucidated. In the present study, a considerable number of NPC cells were found to resist anoikis when grown under anchorage-independent conditions. The cells that resisted anoikis exhibited higher migratory and invasive properties compared with the cells that were cultured under anchorage-dependent condition. These highly migratory NPC cells may survive from anchorage-independent cell cultures and exhibit anoikis resistance compared with the poorly migratory NPC cell line. These results suggest that anoikis resistance is an important characteristic of more aggressive NPC cells.

Previous studies have demonstrated the involvement of STAT3 in cellular growth signaling and its important role in cell survival, proliferation and invasion $(18,19)$. STAT3 has attracted significant attention, as it is involved in malignant progression, and constitutes an attractive therapeutic target. In addition, several studies have demonstrated that STAT3 may induce anoikis resistance, promoting tumor cell survival in certain cancers (20-22). Our results also revealed that anoikis-resistant NPC cells exhibited significantly increased expression of STAT3 with enhanced expression of Bcl-2 and survivin compared with adherent cells.

The present study proposed the hypothesis that the activation of STAT3 induces anoikis resistance in NPC cells and promotes metastasis. We examined the expression of STAT3 in anoikis-resistant tumor cells, and assessed whether inhibition of STAT3 leads to sensitization of NPC cells to anoikis. Following Stattic treatment, significant reduction in anoikis resistance was observed in TW01 and TW06 cells. Moreover, a remarkable reduction in anoikis resistance was observed following STAT3 silencing. The level of STAT3 silencing was significantly correlated with non-adherent cell survival, indicating that anoikis resistance of NPC cells was dependent on STAT3. Notably, STAT3 silencing also reduced the invasiveness and migratory ability of anoikis-resistant NPC cells. The reduction in the rate of invasion was also found to be significantly correlated with the level of STAT3 silencing by shRNAs. These results revealed that the effect of STAT3 on anoikis resistance was correlated with invasion in NPC. In support of the abovementioned findings, there was a significant change in the expression of EMT-related proteins, including $\mathrm{N}$-cadherin and vimentin, which was significantly inhibited after silencing STAT3. Furthermore, knockdown of STAT3 significantly increased E-cadherin expression, as evaluated by western blot analysis. A switch from N-cadherin to E-cadherin expression indicates that STAT3 silencing reverses EMT, and may exert a significant inhibitory effect on the progression of NPC. Decreased CD44 expression was detected by flow cytometry in anoikis-resistant NPC cells following STAT3 knockdown. Previous studies demonstrated that CD44 promotes EMT and is associated with cancer invasion through several mechanisms $(23,24)$. Our results further revealed that STAT3 represents a link between anoikis suppression and EMT, both of which may contribute to metastasis. Since increased EMT-related characteristics have been found to be associated with worse outcomes in patients with $\operatorname{NPC}(25,26)$ these data support that the constitutive activation of STAT3 is predictive of poor prognosis. These findings are in agreement with previous studies investigating the role of STAT3 in the aggressiveness of various tumors $(27,28)$. The important role of STAT3 in conferring resistance to anoikis and promoting metastasis in NPC was further investigated. In conclusion, the present study elucidated the association of STAT3-mediated anoikis resistance with enhanced cell migration and invasion in NPC. The findings suggest that targeting STAT3 may be an important therapeutic strategy for preventing or inhibiting anoikis sensitization and cellular invasiveness in NPC.

\section{Acknowledgements}

This study was supported by a grant from the Taipei City Hospital (TPCH-105-025) and the Department of Health, Taipei City Government, Taipei, Taiwan.

\section{References}

1. Yeh SA, Tang Y, Lui CC, Huang YJ and Huang EY: Treatment outcomes and late complications of 849 patients with nasopharyngeal carcinoma treated with radiotherapy alone. Int J Radiat Oncol Biol Phys 62: 672-679, 2005.

2. Sun JD, Chen CZ, Chen JZ, Li DS, Chen ZJ, Zhou MZ and Li DR: Long term outcomes and prognostic factors of n0 stage nasopharyngeal carcinoma: A single institutional experience with 610 patients. Asian Pac J Cancer Prev 13: 2101-2107, 2012.

3. Nagaprashantha LD, Vatsyayan R, Lelsani PC, Awasthi S and Singhal SS: The sensors and regulators of cell-matrix surveillance in anoikis resistance of tumors. Int J Cancer 128: 743-752, 2011.

4. Zhong X and Rescorla FJ: Cell surface adhesion molecules and adhesion-initiated signaling: Understanding of anoikis resistance mechanisms and therapeutic opportunities. Cell Signal 24: 393-401, 2012.

5. Morimoto-Tomita M, Ohashi Y, Matsubara A, Tsuiji M and Irimura T: Mouse colon carcinoma cells established for high incidence of experimental hepatic metastasis exhibit accelerated and anchorage-independent growth. Clin Exp Metastasis 22: $513-521,2005$.

6. Egwuagu CE: STAT3 in $\mathrm{CD}^{+} \mathrm{T}$ helper cell differentiation and inflammatory diseases. Cytokine 47: 149-156, 2009.

7. Konnikova L, Simeone MC, Kruger MM, Kotecki M and Cochran BH: Signal transducer and activator of transcription 3 (STAT3) regulates human telomerase reverse transcriptase (hTERT) expression in human cancer and primary cells. Cancer Res 65: 6516-6520, 2005.

8. Chen Z and Han ZC: STAT3: A critical transcription activator in angiogenesis. Med Res Rev 28: 185-200, 2008. 
9. Peyser ND and Grandis JR: Critical analysis of the potential for targeting STAT3 in human malignancy. Onco Targets Ther 6: 999-1010, 2013.

10. Banerjee $\mathrm{K}$ and Resat $\mathrm{H}$ : Constitutive activation of STAT3 in breast cancer cells: A review. Int J Cancer 138: 2570-2578, 2016.

11. Weerasinghe P, Garcia GE, Zhu Q, Yuan P, Feng L, Mao L and Jing N: Inhibition of Stat3 activation and tumor growth suppression of non-small cell lung cancer by G-quartet oligonucleotides. Int J Oncol 31: 129-136, 2007.

12. Lui VW, Wong EY, Ho Y, Hong B, Wong SC, Tao Q, Choi GC, $\mathrm{Au}$ TC, Ho K, Yau DM, et al: STAT3 activation contributes directly to Epstein-Barr virus-mediated invasiveness of nasopharyngeal cancer cells in vitro. Int J Cancer 125: 1884-1893, 2009.

13. Wang Z, Luo F, Li L, Yang L, Hu D, Ma X, Lu Z, Sun L and Cao Y: STAT3 activation induced by Epstein-Barr virus latent membrane protein 1 causes vascular endothelial growth factor expression and cellular invasiveness via JAK3 And ERK signaling. Eur J Cancer 46: 2996-3006, 2010.

14. Bensouda Y, Kaikani W, Ahbeddou N, Rahhali R, Jabri M, Mrabti H, Boussen $\mathrm{H}$ and Errihani $\mathrm{H}$ : Treatment for metastatic nasopharyngeal carcinoma. Eur Ann Otorhinolaryngol Head Neck Dis 128: 79-85, 2011.

15. Pan CC, Lu J, Yu JR, Chen P, Li W, Huang ZL, Zhao M, Huang ZM, Xia YF, Wu YH, et al: Challenges in the modification of the M1 stage of the TNM staging system for nasopharyngeal carcinoma: A study of 1027 cases and review of the literature. Exp Ther Med 4: 334-338, 2012.

16. Tan K, Goldstein D, Crowe P and Yang JL: Uncovering a key to the process of metastasis in human cancers: A review of critical regulators of anoikis. J Cancer Res Clin Oncol 139: 1795-1805, 2013.

17. Paoli P, Giannoni E and Chiarugi P: Anoikis molecular pathways and its role in cancer progression. Biochim Biophys Acta 1833: 3481-3498, 2013

18. Raptis L, Arulanandam R, Vultur A, Geletu M, Chevalier S and Feracci H: Beyond structure, to survival: Activation of Stat3 by cadherin engagement. Biochem Cell Biol 87: 835-843, 2009.

19. Fitzgerald JS, Poehlmann TG, Schleussner E and Markert UR: Trophoblast invasion: The role of intracellular cytokine signalling via signal transducer and activator of transcription 3 (STAT3). Hum Reprod Update 14: 335-344, 2008.
20. Zhang W, Zong CS, Hermanto U, Lopez-Bergami P, Ronai Z and Wang LH: RACK1 recruits STAT3 specifically to insulin and insulin-like growth factor 1 receptors for activation, which is important for regulating anchorage-independent growth. Mol Cell Biol 26: 413-424, 2006.

21. Cheng HL, Su SJ, Huang LW, Hsieh BS, Hu YC, Hung TC and Chang KL: Arecoline induces HA22T/VGH hepatoma cells to undergo anoikis - involvement of STAT3 and RhoA activation. Mol Cancer 9: 126, 2010.

22. Hu Y, Chen H, Duan C, Liu D, Qian L, Yang Z, Guo L, Song L, $\mathrm{Yu} \mathrm{M}, \mathrm{Hu} \mathrm{M}$, et al: Deficiency of Erbin induces resistance of cervical cancer cells to anoikis in a STAT3-dependent manner. Oncogenesis 2: e52, 2013.

23. Gao Y, Ruan B, Liu W, Wang J, Yang X, Zhang Z, Li X, Duan J, Zhang F, Ding R, et al: Knockdown of CD44 inhibits the invasion and metastasis of hepatocellular carcinoma both in vitro and in vivo by reversing epithelial-mesenchymal transition. Oncotarget 6: 7828-7837, 2015.

24. Lin CH, Hung PH and Chen YJ: CD44 is associated with the aggressive phenotype of nasopharyngeal carcinoma through redox regulation. Int J Mol Sci 14: 13266-13281, 2013.

25. Luo W and Yao K: Molecular characterization and clinical implications of spindle cells in nasopharyngeal carcinoma: A novel molecule-morphology model of tumor progression proposed. PLoS One 8: e83135, 2013.

26. Lin JC, Liao SK, Lee EH, Hung MS, Sayion Y, Chen HC, Kang CC, Huang LS and Cherng JM: Molecular events associated with epithelial to mesenchymal transition of nasopharyngeal carcinoma cells in the absence of Epstein-Barr virus genome. J Biomed Sci 16: 105, 2009.

27. Kamran MZ, Patil P and Gude RP: Role of STAT3 in cancer metastasis and translational advances. BioMed Res Int 2013: 421821, 2013

28. Devarajan E and Huang S: STAT3 as a central regulator of tumor metastases. Curr Mol Med 9: 626-633, 2009. 